\title{
Effect of Culture on the Management of a Pregnant Fulani Woman with Ovarian Cyst: A Case Report
}

\author{
S.K. Hembah-Hilekaan*
}

Department of Obstetrics and Gynaecology, Benue State University Teaching Hospital, Makurdi, Nigeria

\begin{abstract}
Interactions between clinicians and patients towards pain assessment and management can present a problem due to some differences in perceptions between orthodox medicine and lay beliefs and practices seen in different cultural groups. The Fulani people found in Northern Nigeria and all over West Africa present a unique group in cultural pain perception, in both the male and women folk. This is the report on a case of a large $(>24 \mathrm{~cm})$ right ovarian cyst seen in a sixteen-year-old teenage woman of Fulani descent, presenting with abdominal pain in her sixth month of pregnancy. She had right cystectomy without conservation of the ovary on that side. Histopathological diagnosis was serous cystadenoma. She however, had abortion on the third postoperative day without any warning signs of labour. Although postoperative abortions may be seen at this stage, they are more common in the first trimester of pregnancy.

The early diagnosis and treatment or follow-up of ovarian cystic lesions is necessary for the detection of malignant changes (2\% to $5 \%$ of cysts) and the prevention of other complications involving either the ovary or foetus, in the presence of pregnancy. However, the culture of the Fulani that forbids the expression of pain may complicate the early diagnosis and treatment of both benign and malignant ovarian disease and other similar conditions seen in and out of pregnancy. The objective of this paper is therefore, to help practising clinicians and oncologists, especially Obstetricians and Gynaecologists, towards the early detection, diagnosis and treatment of these conditions by paying attention to both verbal and non-verbal communication of pain, when dealing with this and other monocultural group of people. This may also serve as the basis for further research on cancer and non-cancer pain management among different cultures in our environment.
\end{abstract}

Keywords: Culture, Pain, Fulani, Ovarian, Cyst, Cystectomy, Postoperative, Abortion, Benign, Malignant.

\section{INTRODUCTION}

The ovaries are the site of a wide variety of cysts and tumours, both benign and malignant in and out of pregnancy. The majority of cysts are functional. Routine ultrasonography at 12 to 18 weeks demonstrates ovarian masses in $0.6 \%$ ( 5 to 6 per $1000)$ of pregnancies [1]. The most common size is 7 to $11 \mathrm{~cm}$, and $27 \%$ are larger than $10.5 \mathrm{~cm}$ [2]. The majority present in pregnant than in non pregnant women [3]. Fortunately, only $2 \%$ to $5 \%$ of these cysts are malignant [4]. Although removal may be indicated on the basis of size or possible malignancy, it is usually not an urgent matter [2]. The common presenting symptom of enlarged ovaries during emergency is pain, which may occur due to torsion, rupture, infection or bleeding into the cyst cavity. Torsion seems to occur more often in pregnancy among women in their early reproductive years. Approximately $20 \%$ of cases of torsion occur during pregnancy [5-6]. Although culdocentesis and paracentesis are no more used, they may become necessary in the presence of intraperitoneal bleeding, especially in the developing countries. Laparoscopy on the other hand is an invaluable diagnostic tool in early pregnancy and has become a gold standard for diagnosis of many gynaecologic conditions.

*Address corresponding to this author at the Department of Obstetrics and Gynaecology, Benue State University Teaching Hospital, Makurdi, Nigeria; Tel: +234-8035722335; E-mail: hemhilsk@yahoo.com
The risk of spontaneous abortion during surgery varies according to the gestational age, as the earlier the gestation, the higher the chance of spontaneous abortion. If elective surgery most be performed during pregnancy, the period between 13 to 23 weeks is optimal as the uterus is less sensitive to the stimulating effects of surgery, and the risk of preterm labour is minimal [2]. Preterm deliveries in our environment before 28 weeks are still considered as abortions in the absence of level 3 perinatal centres seen commonly in the advanced world.

Preterm labour/delivery may be the cause of abdominal pain or a consequence of nonobstetrics disease, the diagnosis and treatment of which may be difficult especially among pregnant peri-operative women. Before making a definitive diagnosis, the many obstetrics conditions that pose greater fetal challenge/risk such as chorioamnionitis and abruptio placenta must be considered. In some cultures such as the Fulani's, solitary and unassisted births are valued and seen as a source of pride. This is the report of a case of a huge ovarian cyst seen in the second trimester of pregnancy of a 16 years old teenage Fulani girl, who was admitted in the emergency Gynaecological unit of Sokoto Specialist Hospital, with abdominal pain. She had right ovarian cystectomy after a failed attempt at conservative management with consequent premature delivery. 
The provision of pain relief in labour varies in different cultures, with some avoiding analgesia altogether while others do not want to feel pain at all in labour [7]. The Fulani people found in West Africa present a unique group, in cultural pain perception in both men and women. Although a literature search has failed to give any information on this potentially highrisk behaviour, in relation to abortion or other illnesses except, in delivery; the culture of the Fulani people of Northern Nigeria that forbids the expression of pain as a mark of self discipline may complicate, and even exacerbate the early diagnosis and treatment of both benign and malignant ovarian disease, including other similar conditions seen in women at this stage. The objective of this paper is therefore, to help practising clinicians and oncologists, especially Obstetricians and Gynaecologists, towards the early detection, diagnosis and treatment of these conditions by paying attention to both verbal and non-verbal communication of pain, when dealing with this and other monocultural group of people. This may also serve as a basis for further research on cancer and non-cancer pain management in our environment.

\section{CASE REPORT}

A 16 year-old, gravida 2, Para 1 teenage woman was seen at 6 months gestation at the emergency unit of Sokoto Specialist Hospital in North western Nigeria, and admitted into the Obstetrics and Gynaecology ward following persistent complaint of bouts of severe right- sided pelvic pain of 4 days duration. The pain was of gradual onset initially lasting for a short duration, but this later became severe, with prolonged episodes. Although she did not register for antenatal care, she had noticed that the index pregnancy was progressively bigger than the previous one. There was no associated vagina bleeding, fever; cough or vomiting and her appetite remained good. She had normal bowel movement, with no urinary symptoms and there was no history of weight loss or jaundice. Her last delivery was at home, without incident. She had menarche at 14 years with regular monthly menstrual cycles of 3 days duration.

On review, she was found to be afebrile and stable with a pulse rate of 98 beats/minute and blood pressure of $110 / 80 \mathrm{mmhg}$. The major findings were in the abdomen with a symphysio-fundal height of $40 \mathrm{~cm}$ which was greater than her dates. The abdomen was fluctuant with a fluid thrill. The fetal heart sounds were $160 /$ minute but the parts were difficult to palpate. She had moderate suprapubic tenderness with guarding which was more on the right side. No contractions were felt. On bimanual pelvic examination, the cervix was uneffaced and closed with no additional significant findings. Abdominal ultrasonography confirmed a huge right- side unilocular ovarian cyst $(>24 \mathrm{~cm}$ in diameter) in a pregnant woman, with a normal active 24 week intrauterine foetus. Computerized tomography was not considered on safety grounds and Magnetic resonance imaging test was not available in our hospital. The cervix was closed and measured $4.0 \mathrm{~mm}$ in diameter.

She was placed on analgesics and counselled for possible conservative management on the ward. She however, had laparotomy on the second day of admission as the operation of choice due to increasing abdominal pain, without any sign of labour as well as, the combined size of the pregnant uterus and cyst. A huge right ovarian cyst containing about $8 \mathrm{~L}$ of clear serous fluid was removed (after partial aspiration due to the difficulty in delivering the cyst) through a midline longitudinal incision which was extended above the umbilicus. There was no ascites but an aspirate of peritoneal fluid washing was taken. No ovarian tissue was identifiable on the involved side. The base of the cyst was found to be haemorrhagic; and it was mobilised, ligated and cut. There was minimal tumour spill into the peritoneal cavity with adequate lavage done. The washing, cyst wall and contents were sent for histology. She was given antibiotics and analgesics for adequate pain relief for one week duration, and was also told to report any sign of labour. On the second postoperative day, she expelled a live male abortus with minimal signs of labour. The fetus and membranes weighed about 700 grammes. The foetus died 15 minutes later. She had an uneventful recovery and was discharged home on the $7^{\text {th }}$ postoperative day after all sutures were removed.

The histology result showed a fibro-collagenous cyst wall lined by tall columnar cells with basal nuclei. There was no evidence of malignancy. This confirmed the diagnosis of serous cystadenoma. She was lost to follow-up in the out-patient clinic after one visit.

\section{DISCUSSION}

The Fulani people are a group of nomads found all over West Africa but in Nigeria, they are mainly seen in the northern part of the country. An important feature of the behavioural code of the Fulani's is self discipline and the avoidance of expression of emotion or pain [8]. The Fulani culture forbid open expression of pain especially among their male folk as is exhibited by 
open beating with sticks across the chest by young men who must not show any sign of pain. Women on the other hand, must resist open demonstration of pain, during child birth. This may have accounted for the delayed presentation and postoperative absence of obvious signs of labour in this woman.

Ovarian cysts as seen in this Fulani teenager are fluid-filled sacs found within the ovaries. They are usually benign and may be seen from the neonatal life up to the period of the menopause. Most ovarian cysts occur during infancy and adolescence, which are hormonally active periods of development [9]. Approximately $50 \%$ of ovarian tumours are benign epithelial tumours [10]. The presentation of this 16 years old woman fits into exactly the active hormonal phase of development enunciated above. Benign ovarian cysts even though may be very large, cause relatively few symptoms. Abdominal distension is the most common presenting symptom. This may cause pressure symptoms such as oedema and hydroureters/hydronephrosis with wasting symptoms in some instances, although, none was seen in this case.

Abdominal pain in the female may present diagnostic dilemma and in the Fulani's, may even be more problematic. The spectrum of pain as a disease in women is wide involving all age groups. This can be from simple benign cystic conditions that disappear with monitoring to life threatening diseases like appendicitis and ruptured ectopic pregnancies. Pain may also be seen as a result of torsion, tension, rupture, haemorrhage, infection and even malignant changes within the tumour mass. Additionally, in pregnancy, this may be due to labour, abruption or other complications of pregnancy. The observation of signs of inflammation at the base of the cyst intraoperatively was an early sign of torsion and possible detorsion.

Among the Fulani's, pregnancies are kept secret for as long as possible, and traditionally the mother delivers alone or calls a relative, often her own mother to cut the cord [11]. This was clearly demonstrated in this native woman who delivered alone without any assistance or sign of pain even while in hospital. This complication (premature labour) could have been anticipated and possibly avoided with the early additional use of tocolytics to complement the analgesia. Other approaches to minimize the risk of premature labour include uterine monitoring during surgery and the immediate postoperative period for early detection of regular uterine contractions, the use of regional anaesthesia to decrease postoperative pain and subsequent release of catecholamine's which can stimulate contractions, and continued infusion of narcotics for up to 72 hours.

There are several factors responsible for the disparities in pain perception and care; these may be biological, cultural, personal beliefs and social factors. Other related issues include pain expression, pain language, lay remedies, social role and expectations, and perceptions of the medical care system. The lack of adequate pain assessment and disagreement in pain intensity are major causes of persistent undertreated pain especially when the patient is of a different culture or language from the care provider. Together, these may have contributed in this patient. Although, disagreements in pain rating and treatment between patients and physicians is not new, the current opinion supports the assertion of many organisations that providers should accept patient's self-rating of pain, measured by the 11-point numeric rating scale, for which scores range from 0 (no pain) to 10 (unbearable pain).

The histology confirmed the diagnosis of benign serous cystadenoma. Ultrasonographic features most often used to distinguish benign from malignant ovarian masses include size, number of loculations, and presence of papillary excrescences, overall density and pulsatility. Generally, unilocular cysts without internal echoes of less than $5 \mathrm{~cm}$ in diameter, the likelihood of malignancy is low and expectant management is acceptable as was seen here. Multilocular masses with solid components, $70 \%$ are malignant [12]. Ultrasound guided tumour aspiration may be used to avoid open laparotomy in some cases; however recurrence is high, and examination of tumour fluid has low sensitivity [12]. Another important problem is the impact of tumour spill in the management of early ovarian cancer that is undiagnosed at the time of surgery $[12,13]$. Studies of patients with stage 1 tumours however, show that intraoperative rupture/spill as may have occurred in this case does not worsen prognosis whereas preoperative spontaneous rupture or ascites does [12].

Other modalities of treatment apart from conservative management and open surgery include laparoscopy which could not be done here due to the size of both the cyst and pregnancy.

This article discusses the negative influence of culture on pain limiting the early presentation, diagnosis and treatment or possible follow-up of both 
benign and malignant ovarian lesions and their complications, especially in the presence of pregnancy. It is true that other factors endemic in our environment such as poverty and ignorance, preference for home deliveries, dearth of infrastructure and personnel and outright discrimination may have limited the early presentation of this nomadic Fulani woman. Further studies, could improve cultural competence and communication in the clinical encounters to reduce disagreements, improve outcome, and reduce gaps in cultural pain perception and management of these and related cases towards a more favourable outcome. This should serve as a guide to practising physicians, oncologists and Obstetricians and Gynaecologists when faced with women presenting in similar circumstances.

\section{REFERENCES}

[1] Hogston P, Lifford RJ. Ultrasound study of ovarian cysts in pregnancy: Prevalence and significance. $\mathrm{Br} \mathrm{J}$ Obstet Gynecol 1986; 93: 625. http://dx.doi.org/10.1111/j.1471-0528.1986.tb08037.x

[2] Newton, E, Glob. libr. women's med., (ISSN: 1756-2228) 2008; DOI 10.3843/GLOWM. 10191

[3] Lee $\mathrm{CH}$, Raman S, Sivanesaratman V. Torsion of ovarian tumours: a clinicopathological study. Int J Gynaecol Obstet 1989; 28: 21-25 http://dx.doi.org/10.1016/0020-7292(89)90539-0
[4] Goldman NA, Lee M, Priore G. Surgical Problems: management of the Obstetric patient with malignancy. http://www.glowm.com/index.html?p=glowm.cml/section_vie w\&articleid=193 Under review- Update due 2012.

[5] Bider D, Mashiach S, Dulitzky M, Kokia E, Lipitz S, BenRafael Z. Clinical, surgical and pathologic findings of adnexal torsion in pregnant and nonpregnant women. Surg Gynecol Obstet 1991; 173(5): 363-6. [Medline].

[6] Griffin D, Shiver SA. Unusual presentation of acute ovarian torsion in an adolescent. Am J Emerg Med 2008; 26(4): 520.e1-3. [Medline].

[7] Audu B, Yahaya U, Bukar M, El-Nafaty A, Abdullahi H, Kyari O. Desire for pain relief in labour in Northern Nigeria. J. Public Health Epidermiol 2009; 1(2): 053-057. Available online at http://www.acedamicjournals-org/jphe

[8] Kirk-Greene AHM. Survival and symbiosis. In: Pastoralist of the West African savannah,adamu M, Kirk-Greene AHM eds. Manchester, Manchester University Press 1986.

[9] Walter WV, Silverberg MA, Aronson AA. Ovarian cyst in emergency http://emedicine.medscape.com/article/795877overview\#showall Updated: Jul 19, 2010.

[10] Maclean AB. Benign disease of vagina, cervix and ovary. In Dewhurst's Textbook of obstetrics and Gynaecology for Postgraduates. Keith ED, Ed. $6^{\text {th }}$ ed. Blackwell Science Ltd. 1999; pp. 582-589.

[11] Dehne KL, Wacker J, Cowley J. Training birth attendants in the Sahel. World Health Forum 1995; 16: 415-19.

[12] Roger PS. Benign disorders of the ovaries. In: Precis. An Update in Obstetrics and Gynecology $2^{\text {nd }}$ ed. American College of Obstetricians and Gynecologists 2001; pp. 86-90.

[13] Disaia PJ, Creasman WT. The adnexal mass and early ovarian cancer. In: Clinical Gynaecologic Oncology. $5^{\text {th }}$ ed. St. Louis Mosby 1997; pp. 253-281.

(C) 2012 S.K. Hembah-Hilekaan; Licensee Lifescience Global.

This is an open access article licensed under the terms of the Creative Commons Attribution Non-Commercial License (http://creativecommons.org/licenses/by-nc/3.0/) which permits unrestricted, non-commercial use, distribution and reproduction in any medium, provided the work is properly cited. 